TRANSACTIONS OF THE

AMERICAN MATHEMATICAL SOCIETY

Volume 180, June 1973

\title{
BARYCENTERS, PINNACLE POINTS, AND DENTING POINTS
}

BY

\author{
SUR JIT SINGH KHURANA
}

\begin{abstract}
Some properties of probability measures, on closed convex bounded sets in locally convex spaces, having barycenters are obtained. Also some geometric and measure-theoretic characterizations of pinnacle points are given, and a result about denting points is proved.
\end{abstract}

Introduction and notations. Let $X$ be a bounded, closed, convex subset of a Hausdorff locally convex space $E$ over reals, $C_{b}(X)$ (resp., $C(X)$ ) all bounded real-valued (resp., all real-valued) continuous functions on $X, M(=M(X))$ the set of all positive linear functionals on $C_{b}(X)$, each taking the value 1 on the constant function 1 ( $M$ is also the set of all nonnegative, regular, finitely additive measures, each of a total mass 1 , on the algebra O generated by zero-sets, i.e., the sets of the form $f^{-1}\{0\}$ for $\left.f \in C_{b}(X),[16]\right) . M_{\sigma}, M_{\tau}$ and $M_{t}$ are, respectively, the countably additive, $r$-additive and tight elements of $M$ ([4], [9], [14], [15], [16]). Every element $\mu \in M_{\tau}$ has a unique extension to a regular Borel measure on $X$ which will also be denoted by $\mu$ ([9], [14]). We will always take the weak topology on $M$; in this topology $\mu_{\alpha} \rightarrow \mu$ if and only if $\mu_{\alpha}(g)$ converges to $\mu(g)$ for every $g \in C_{b}(X)$. $M$ is compact in this topology.

A point $x \in X$ is called the barycenter of a $\mu \in M$, written as $\beta(\mu)=x$, if $\mu(f \mid x)=f(x)$, for every $f \in E^{\prime}$, the topological dual of $E$ ([2], [3], [10]). As in [1, p. 15] we define an equivalent relation ' $~ \sim$ ' in $X$ : for $x \in X, y \in X$, we say $x \sim y$ if there exists $r>0$ such that $x+r(x-y)$ and $y+r(y-x)$ are both in $X$. This relation partitions $X$ into disjoint convex subsets, called parts of $X$.

For any $f \in C_{b}(X)$, we define $\bar{f}(x)=\operatorname{Inf}\left\{g(x): g \geq f\right.$ and $\left.g \in\left(E^{\prime}+R\right) \mid X\right\}$ for every $x \in X$. Since any bounded affine function $b \in C_{b}(X)$ has the property that $b(x)=$ $\operatorname{Inf}\left\{k(x): k \geq b, k \in\left(E^{\prime}+R\right) \mid X\right\}[6$, II, p. 222] we also have $\bar{f}(x)=\operatorname{Inf}\{g(x): g \geq f$, $g$ affine, $\left.g \in C_{b}(X)\right\}[2$, p. 335]. It is easy to see that $\bar{f}$ is bounded, concave, upper semicontinuous, and $\bar{f}(x)=\operatorname{Sup}\{\mu(f): \mu \in M: \beta(\mu)=x\}$ ([2, p. 335], [10, pp. 19-21]). We will denote the set of real numbers by $R$, and the set of positive integers by $N$. The elements of $M=M(X)$ will also be called probability measures

Received by the editors April 25, 1972 and, in revised form, October 27, 1972. AMS (MOS) subject classifications (1970). Primary 46B99; Secondary 28 A40.

Key words and phrases. Closed bounded sets, measures and barycenters of measures, $\tau$-smooth measures, tight measures, pinnacle points, part of a convex set, denting points. 
on $X$. Nets and subnets are taken in the sense of [5]. Sometimes instead of saying ' $x$ is the barycenter of $\mu$ ', we shall say ' $\mu$ represents $x$ '.

$\S 1$ contains some results about the measures having barycenters. In $\$ 2$, some theorems of [2] regarding pinnacle points are generalized and sharpened. $\$ 3$ contains a much simpler proof of a strengthened form of an integral representation theorem of [2, Proposition 2.6, p. 332].

1. In this section, $X$ is always a closed convex bounded subset of a Hausdorff locally convex space $E$ over reals, and $C_{b}(X)$ and $M$ have the meanings explained in the introduction.

Theorem 1.1. Let $X$ be a complete, convex, and bounded subset of $E$ and $\mu \in M_{\tau}$, baving $x \in X$ as its barycenter. Then there exists a net $\left\{\mu_{\alpha}\right\}(\alpha \in I)$ of discrete probability measures, each baving $x$ as its barycenter, converging to $\mu$ weakly.

Proof. It is proved in [7] that every element of $M_{\tau}$ will have a barycenter in this case. Now proceeding exactly as in [6, II, Theorem 9, p. 228] we get the result.

Theorem 1.2. Let $X$ be a complete, convex, and bounded subset of $E, \mu \in M_{\tau}$ baving $x \in X$ as its barycenter, and $f$ a semicontinuous, bounded, affine function on $X$. Then $\int f d \mu=\mu(f)=f(x)$.

Proof. Let $f$ be u.s.c., and affine and bounded. Proceeding as in [6, II, Theorem 1, p. 222], $f(y)=\operatorname{Inf}\left\{g(y)+r: g \in E^{\prime}, r \in R, g+r \geq f\right.$ on $\left.X\right\}$, for every $y \in X$. From this it follows that $\mu(f) \leq f(x)$ and $f=\operatorname{Inf} b_{\gamma}$ (pointwise), where $\left(b_{\gamma}\right)_{\gamma \in J}$ are the pointwise inf of the finite collection of bounded continuous affine functions dominating $f$ and thus are monotonically decreasing. Since $\mu \in M_{\tau}, \mu(f)=$ $\lim \mu\left(b_{\gamma}\right)$ ([14], [16]). By Theorem 1.1, $\mu=\lim _{\alpha \in I} \mu_{a}, \mu_{\alpha}$ discrete probability measures, and $\beta\left(\mu_{\alpha}\right)=x, \forall \alpha$, and so $\mu_{\alpha}\left(b_{\gamma}\right) \geq \mu_{\alpha}(f)=f(x), \forall \alpha$ and $\forall \gamma$, which implies that $\mu\left(b_{\gamma}\right) \geq f(x) \forall y$ and so $\mu(f) \geq f(x)$. This proves the result.

Remark 1. Proposition 1.11 of [2, p. 327] is a particular case of this theorem.

Remark 2. Theorem 1.1 cannot hold for every $\mu \in M$. An example to this effect is given in [2, p. 339].

2. In this section we derive some geometrical and measure-theoretic properties of pinnacle points. Also, a result about denting points is proved.

Let $E^{\prime}, E^{\prime \prime}$ be respectively the dual and bidual of a Hausdorff locally convex space $E$ over reals, $X$ a convex $\sigma\left(E^{\prime}, E^{\prime \prime}\right)$-closed and bounded subset of $E^{\prime}$, and $\tau_{0}, \tau, \tau_{1}$ the topologies induced on $X$ by $\sigma\left(E^{\prime}, E\right)$, strong, $\sigma\left(E^{\prime}, E^{\prime \prime}\right)$ topologies on $E^{\prime}$, respectively [12]. 
Definition 2.1. A point $x \in X$ is called a pinnacle point of $X$ if whenever $\left\{x_{a}\right\}_{a \in I},\left\{y_{\alpha}\right\}_{a \in I}$ are nets in $X$ such that $\left\{1 / 2\left(x_{a}+y_{\alpha}\right)\right\}_{a \in I} \tau_{1}$-converges to $x$, then $x \in \tau_{0}-\operatorname{cl}\left[\left\{x_{a}\right\}_{a \in I} \cup\left\{y_{a}\right\}_{a \in I}\right][2, \mathrm{p} .335]$.

We denote by $X_{0}$ the closure of $X$ in the completion of $\left(E^{\prime}, \sigma\left(E^{\prime}, E\right)\right)$, by $w_{0}$ the topology induced by this completion on $X_{0}$, by $X_{1}$ the closure of $X$ in the completion of $\left(E^{\prime}, \sigma\left(E^{\prime}, E^{\prime \prime}\right)\right)$, and by $w_{1}$ the topology induced on $X_{1}$ by this completion. Then $\tau_{1}=w_{1 \mid X}$ and $\tau_{0}=w_{0 \mid X}$. It is easy to see that $\left(X_{0}, w_{0}\right)$ and $\left(X_{1}, w_{1}\right)$ are convex and compact.

Lemma 2.1. Let $P, Q$ be two subsets of $X$, baving disjoint closures in $\left(X_{0}, w_{0}\right)$. Then $P$ and $Q$ bave disjoint closures in $\left(X_{1}, w_{1}\right)$.

Proof. Suppose not. Then there exist nets $\left\{x_{a}\right\} \subset P,\left\{x_{a}\right\} \subset Q(\alpha \in I)$ such that $\left\{x_{a}-y_{a}\right\}_{a \in I}$ converges to 0 in $\left(E^{\prime}, \sigma\left(E^{\prime}, E^{\prime \prime}\right)\right)$. Thus $\left\{x_{a}-y_{a}\right\}_{a \in I}$ converges to 0 in $\left(E^{\prime}, \sigma\left(E^{\prime}, E\right)\right)$. This implies that $x=y$ for some cluster point $x$ of $\left\{x_{a}\right\}_{a \in I}$ and some cluster point $y$ of $\left\{y_{a}\right\}_{a \in I}$ in $\left(X_{0}, w_{0}\right)$, due to the compactness of $\left(X_{0}, w_{0}\right)$, a contradiction.

Let $T$ be the part of $X_{1}$ which contains $x_{0} \in X$, and $\bar{T}=w_{1}-\operatorname{cl}(M)$.

Theorem 2.2. For a point $x_{0} \in X$, the following statements are equivalent.

(i) $x_{0}$ is a pinnacle point of $X$.

(ii) For any positive integer $n$, and $\left\{\lambda_{i}\right\}_{1 \leq i \leq n}, 0<\lambda_{i} \leq 1, \sum_{i=1}^{n} \lambda_{i}=1$, whenever $\left\{y_{a}^{(i)}\right\}_{a \in I}(1 \leq i \leq n)$ are nets in $X$ for which $\sum_{i=1}^{n} \lambda_{i} y_{a}^{(i)} \tau_{1}$-converges to $x_{0}$ then $x_{0} \in \tau_{0}-\operatorname{cl} \bigcup_{i=1}^{n}\left\{y_{a}^{(i)}\right\}_{a \in I}$.

(iii) For any $\tau_{0}^{-x} 0^{-n b d} V$ in $X$, the $w_{1}^{-c l}(V)$ in $X_{1}$ contains $T$.

Proof. Evidently (ii) $\Rightarrow$ (i). We first prove that (i) $\Rightarrow$ (iii). Suppose (iii) does not hold. Then $\exists$ a $\tau_{0}$-nbd $G$ of $x_{0}$ such that $\tilde{y} \in T \backslash \bar{G}, \bar{G}$ being the closure of $G$ in $\left(X_{1}, w_{1}\right)$. Thus $\exists \tilde{z} \in X_{1}$, and $\lambda, 0<\lambda<1$, such that $x_{0}=\lambda \tilde{y}+(1-\lambda) \tilde{z}$, which means there exist nets $\left\{y_{a}^{\prime}\right\},\left\{z_{a}^{\prime}\right\},\left\{y_{a}^{\prime}\right\} \subset X \backslash G$ such that $\lambda y_{a}^{\prime}+(1-\lambda) z_{\alpha}^{\prime}$ $\tau_{1}$-converges to $x_{0}$ and so $\left\{z_{a}^{\prime}\right\}$ does not $\tau_{0}$-converge to $x_{0}$. Thus we can find subnets $\left\{y_{y}\right\}_{\gamma \in J},\left\{z_{y}\right\}_{y \in J}$ such that $\left\{y_{\gamma}\right\}_{\gamma \in J} \cup\left\{z_{\gamma}\right\}_{\gamma \in J} \subset X \backslash G_{0}$ for some $\tau_{0} x_{0}$-nbd $G_{0}$. If $\lambda \geq 1 / 2$, let $\tilde{b}=2 x_{0}-\tilde{y} \in X_{1}$ and if $\lambda<1 / 2$, let $\widetilde{k}=2 x_{0}-\tilde{z} \in X_{1}$. In the first case $x_{0}=(\tilde{y}+\tilde{b}) / 2$, and proceeding as above we shall find nets $\left\{y_{\rho}\right\}_{\rho \in \Lambda},\left\{b_{\rho}\right\}_{\rho \in \Lambda}$ in $X$ such that $\left(b_{\rho}+y_{\rho}\right) / 2 \tau_{1}$-converges to $x_{0}$, but $\left\{b_{\rho}\right\}_{\rho \in \Lambda} \cup$ $\left\{y_{\rho}\right\}_{\rho \in \Lambda} \subset X \backslash G_{1}$, for some $\tau_{0}-x_{0}-\mathrm{nbd} G_{1}$, a negation of (i). A similar argument holds for the second case and so (i) does not hold.

Now we shall prove that (iii) $\Rightarrow$ (ii). If (ii) does not hold, with the notations of (ii), $x_{0} \notin \tau_{0}-\mathrm{cl}(P)$ where $P=\bigcup_{i=1}^{n}\left\{y_{a}^{(i)}\right\}_{a \in I}$, and so there exists a $\tau_{0}-x_{0}$-nbd $Q$ of $x_{0}$ such that $P$ and $Q$ have disjoint closures in $\left(X_{0}, w_{0}\right)$. By Lemma 2.1, 
$P$ and $Q$ have disjoint closures in $\left(X_{1}, w_{1}\right)$. Taking subsets and renaming, if necessary, we may assume that $y_{\alpha}^{(i)} w_{1}$-converges to $\tilde{y}^{(i)} \neq x_{0}, \forall i, 1 \leq i \leq n$, and $\sum_{i=1}^{m} \lambda_{i} \tilde{y}^{(i)}=x_{0}$. Thus for any $\eta, 0<\eta<1,(1-\eta) \tilde{y}^{(i)}+\eta x_{0} \in T$ and so $(1-\eta) \tilde{y}^{(i)}+\eta x_{0} \in w_{1}-\mathrm{cl}(Q)$, by (iii), and so $\tilde{y}^{(i)} \in w_{1}-\mathrm{cl}(Q)(1 \leq i \leq n)$; but $\tilde{y}^{(i)} \in w_{1}-\operatorname{cl}(P)$ and so we get a contradiction.

Example 2.3. With the notations introduced in the beginning of $\$ 2$, the following is an example in which $x_{0}$ is a pinnacle point of $X$, but the part $T$, of $X_{1}$, which contains $x_{0}$ is nontrivial.

Let $E$ be the Banach space $c_{0}$ so that $E^{\prime}=l, E^{\prime \prime}=l_{\infty}=C(\beta N)(\beta N$ is the Stone-Čech compactification of the positive integers) and $E^{\prime \prime \prime}=$ (finite regular Borel measures on $\beta N\}$. In $l_{1}$ let $\delta_{k}=(0,0, \cdots, 0,1,0, \cdots)(1$ in the $k$ th place $)$ and let $A=\left\{\delta_{2 n}: n=1,2, \cdots\right\} \cup\left\{-\delta_{2 n}+(1 / n) \delta_{2 n+1}: n=1,2, \cdots\right\}$ and let $X=$ weak $^{*}$ closed convex hull $A$.

$X$ is weak* compact and convex so that each of its extreme points is a pinnacle point. By the Milman converse to the Krĕn-Milman theorem, the extreme points of $X$ are contained in $A \cup\{0\}$. If 0 were not an extreme point of $X$, by the Choquet theorem, it could be written as an infinite convex combination of the points of $A$. Since this is impossible, 0 is an extreme point of $X$. Moreover, if $\chi: l_{1} \rightarrow l_{1}^{\prime \prime}$ is the natural embedding, then $\left(X_{1}, w_{1}\right)$ is affinely homeomorphic to the weak* closure of $\chi(X)$ in $C(\beta N)^{\prime}$. Let $a \in \beta N \backslash N$ be any cluster point of $\{2 n: n=1,2, \cdots\}$. Then $\epsilon_{a}$ to $-\epsilon_{a}$ are points of $X_{1}$, so that each point of the

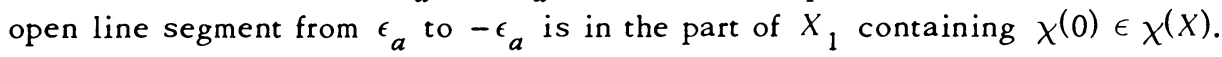
Thus 0 is a pinnacle point of $X$ whose part in $X_{1}$ is nontrivial.

In the next theorem we give some measure-theoretic characterizations of pinnacle points.

Theorem 2.4. With r-topology on $X$, for a point $x_{0} \in X$, the following statements are equivalent.

(i) $x_{0}$ is a pinnacle point of $X$.

(ii) For any probability measure $\nu$ on $\left(X_{1}, w_{1}\right)$ representing $x_{0}, \operatorname{supp}(\nu) \subset$ $w_{1}-\mathrm{cl}(G)$ in $\left(X_{1}, w_{1}\right)$ for every $\tau_{0}-n b d G$ of $x_{0}$ in $X$.

(iii) For any measure $\mu \in M$, representing $x_{0}, S \in \mathfrak{A}, \mu(S)>0$ implies that $x_{0} \in \tau_{0}-\mathrm{cl}(S)$.

(iv) For any measure $\mu \in M$, representing $x_{0}, \mu(g)=g\left(x_{0}\right)$ for any $g \in C_{b}(X)$ which is $\tau_{0}$-continuous.

(v) $\bar{g}\left(x_{0}\right)=g\left(x_{0}\right)$ for any bounded $\tau_{0}$-continuous function on $X$.

Proof. (i) $\Rightarrow$ (ii). Let $\nu$ be a discrete probability measure on $\left(X_{1}, w_{1}\right)$ having $x_{0}$ as its barycenter; then supp $\nu \subset \bar{T}$, and so supp $\nu \subset w_{1}-\operatorname{cl}(G)$ for any $\tau_{0}$-nbd $G$ of $x_{0}$, by Theorem 2.2. If $\nu$ is any probability measure on $\left(X_{1}, w_{1}\right)$ 
having $x_{0}$ as its barycenter, there exists a net $\left(\nu_{\alpha}\right)_{a \in I}$ of discrete probability measure converging to $\nu$ weakly, and $\beta\left(\nu_{a}\right)=x_{0}, \forall a \in I$. Let $Z$ be any zero set in $\left(X_{1}, w_{1}\right), Z \supset T$, then $\nu(Z) \geq \overline{\lim } \nu_{a}(Z)=1[16, \mathrm{p} .182]$ and so $\nu(\bar{T})=1$. Using Theorem 2.2, we prove that (ii) holds.

(ii) $\Rightarrow$ (iii). Let $\mu \in M, \beta(\mu)=x_{0}$. Define a probability measure $\bar{\mu}$ on $\left(X_{1}, w_{1}\right)$ by $\bar{\mu}(g)=\mu\left(g_{X_{X}}\right)$ for every $g \in C\left(X_{1}\right)$. Then $\beta(\bar{\mu})=x_{0}$. Take $S \in$ Ol such that $x_{0} \notin \tau_{0}-\mathrm{cl}(S)$, and let $G$ be a $\tau_{0}$-nbd of $x_{0}$ such that $S$ and $G$ have disjoint closures in $\left(X_{0}, w_{0}\right)$. By Lemma 2.1, $w_{1}-\operatorname{cl}(G) \cap w_{1}-\operatorname{cl}(S)=\varnothing$ in $\left(X_{1}, w_{1}\right)$. Define $g \in C\left(X_{1}\right), g=1$ on $S, g=0$ on $G$, and $0 \leq g \leq 1$. By (ii), $\bar{\mu}$ is supported by $w_{1}-\mathrm{cl}(G)$ and so $\mu(S) \leq\left.\int g\right|_{X} d \mu=\int g d \bar{\mu}=0$, which gives $\mu(S)=0$.

(iii) $\Rightarrow$ (iv). Let $g$ be $\tau_{0}$-continuous, $g\left(x_{0}\right)=0$, and $0 \leq g \leq 1$. Take $\epsilon>0$, and let $G=\{x \in X: g(x)<\epsilon\}$. If $\mu \in M$, such that $\beta(\mu)=x_{0}$, then by (iii), $\int g d \mu=\int_{G} g d \mu \leq \epsilon$, which gives $\mu(g)=g\left(x_{0}\right)$, from which it easily follows that $\mu(b)=b\left(x_{0}\right)$ for any bounded, $\tau_{0}$-continuous function $b$ on $X$.

(iv) $\Rightarrow(\mathrm{v})$. Let $g$ be any bounded, $\tau_{0}$-continuous function on $X$. Then $\bar{g}\left(x_{0}\right)=\operatorname{Sup}\left\{\mu(g)=\mu \in M, \beta(\mu)=x_{0}\right\}=g\left(x_{0}\right)$, by (iv).

(v) $\Rightarrow$ (i). If $x_{0}$ is not a pinnacle point then there are nets $\left\{x_{a}\right\}_{a \in I}$ and $\left\{y_{a}\right\}_{a \in I}$ in $X$ such that $1 / 2 x_{a}+1 / 2 y_{a}$ converges on the $\tau_{1}$ topology to $x_{0}$ and yet $x_{0} \notin B$ where $B=\tau_{0}$-closure $\left(\left\{x_{a}\right\}_{a \in I} \cup\left\{y_{a}\right\}_{a \in I}\right)$. Choose any bounded $\tau_{0}$ continuous function $g$ on $X$ for which $g\left(x_{0}\right)=0$ and $g \mid B \cap X=1$ and $0 \leq g \leq 1$ on $X$. By hypothesis, $\bar{g}\left(x_{0}\right)=g\left(x_{0}\right)$. Since $\bar{g}$ is upper semicontinuous and concave (and since $1 / 2 x_{a}+1 / 2 y_{a}$ converges on the $\tau_{0}$ topology to $x_{0}$ ) we have

$$
\begin{aligned}
0=g\left(x_{0}\right)=\bar{g}\left(x_{0}\right) & \geq \overline{\lim } \bar{g}\left(1 / 2 x_{\alpha}+1 / 2 y_{\alpha}\right) \geq \overline{\lim }\left[1 / 2 \bar{g}\left(x_{\alpha}\right)+1 / 2 \bar{g}\left(y_{\alpha}\right)\right] \\
& \geq \overline{\lim }\left[1 / 2 g\left(x_{\alpha}\right)+1 / 2 g\left(y_{\alpha}\right)\right]=1,
\end{aligned}
$$

a contradiction.

Definition. Let $X$ be a closed convex bounded subset of a Hausdorff locally convex space $E$ over reals. A point $x \in X$ is called a denting point of $X$ if for any neighborhood $V$ of $x, x \notin \overline{\operatorname{conv}(X \backslash V)}$. Choquet calls the se points strongly extreme points [3, Vol. 2, p. 97]. For Banach spaces, Rieffel [12] calls them denting points.

In [8] it is proved that a point $x$ is a denting point if and only if $\epsilon_{x}$ is the only element of $M$ having $x$ as its barycenter. Using this result we prove the following theorem, in which Dent $(X)$ denotes all denting points of $X$.

Theorem 2.5. Let $X$ be a closed, convex bounded subset of a locally convex space $E$ over reals. Then Dent $X=\bigcap\left\{P_{f}: f \in C_{b}(X)\right\}$, where $P_{f}=\{x \in X: \bar{f}(x)=$ $f(x)\}$.

Proof. Suppose $x \in$ Dent $X$, and $f \in C_{b}(X)$, then $\bar{f}(x)=\operatorname{Sup}\{\mu(f): \mu \in M, \beta(\mu)=$ $x\}=f(x)$, since the only $\mu \in M$ having $x$ as its barycenter is $\epsilon_{x}$ [8]. Conversely, 
suppose $x \in X \backslash \operatorname{Dent}(X)$. Then, by [8], there exists a $\mu \in M, \mu \neq \epsilon_{x}$, such that $\beta(\mu)=x$. Define a probability measure $\bar{\mu}$ on $\widetilde{X}$, the Stone-Čech compactification of $X$, by $\bar{\mu}(g)=\mu\left(g_{\mid X}\right), \forall g \in C(\tilde{X})$, and let $\tilde{y} \in \operatorname{Supp} \bar{\mu}, \tilde{y} \neq x$. Take $f_{0} \in C(\tilde{X})$, such that $f_{0}(x)=0, f_{0}(\tilde{y})=1,0 \leq f_{0} \leq 1$, and let $f=f_{0} \mid x$. Then $\mu(f)=\bar{\mu}\left(f_{0}\right)>0$ $=f(x)$, and so $\bar{f}(x)>f(x)$. The result now follows.

3. In this section we generalize and sharpen a Theorem of [2, Proposition 2.6, p. 332] and give a very simple proof.

Theorem 3.1. Let $X$ be a bounded convex subset of a Hausdorff locally convex space $E$ over reals, and assume $X$ is the closed convex bull of its extreme points. Let $\widetilde{X}$ be the Stone-ČCech compactification of $X$. Then for every $x \in X$, there exists a probability measure $\mu$ on $\widetilde{X}$, sucb that $\mu\left(f^{\prime}\right)=f(x), \forall f \in E^{\prime}$ ( $f^{\prime}$ being the unique extension of $f_{\left.\right|_{X}}$ to $\left.\tilde{X}\right)$ and $\mu(P)=0$ for any Borel set $P$, in $\tilde{X}$, which is disjoint from $(\tilde{X} \backslash X) \cup \frac{1}{\operatorname{ext} X}$, where $\overline{\text { ext } X}$ denotes the closure of ext $X$ in $X$. (The topology on $X$ is the one induced by $E$.)

Proof. Take $x \in X$. There exists a net $\left\{x_{a}\right\}_{a \in I}$ in $X$, such that $x_{a} \rightarrow x$ and $x_{\alpha}=\sum_{i=1}^{p_{a}} \lambda_{i}^{(a)} x_{i}^{(a)}\left(0<\lambda_{i}^{(a)} \leq 1, \sum_{i=1}^{p_{a}} \lambda_{i}^{(\alpha)}=1, x_{i}^{(a)}\right.$ being extreme points of $\left.X\right)$. Let $\mu_{0}$, in $M$, be a cluster point of the net $\left\{\mu_{a}\right\}_{a \in I}, \mu_{a}=\sum_{i=1}^{p a} \lambda_{i}^{(\alpha)} \epsilon_{x_{i}^{(\alpha)}}$. Then $\beta\left(\mu_{0}\right)=x$. Define $\mu$, a probability measure on $\tilde{X}$, by $\mu(g)=\mu_{0}(g \mid X)$ for every $g \in C(\tilde{X})$. Then $\mu\left(f^{\prime}\right)=f(x), \forall f \in E^{\prime}$. If $C$ is a compact subset of $\tilde{X}$ disjoint from $(\tilde{X} \backslash X) \cup \overline{\text { ext } X}$, then the closure of ext $X$, in $\tilde{X}$, and $C$ are disjoint compact sets in $\tilde{X}$ and so there exists $b \in C(X)$ such that $b(C)=1, b \equiv 0$ on ext $X$, and $0 \leq b \leq 1$. Now $\mu(C) \leq \mu(b)=\mu_{0}\left(\left.b\right|_{X}\right)=\lim \mu_{\gamma}\left(b_{\mid X}\right)=0\left(\left\{\mu_{\gamma}\right\}\right.$ being a subset of $\left\{\mu_{a}\right\}$ such that $\mu_{\gamma} \rightarrow \mu_{0}$ ), which gives $\mu(C)=0$. By regularity of $\mu, \mu(P)=0$ for any Borel set $P$, in $\tilde{X}$, disjoint from $(\tilde{X} \backslash X) \cup \overline{\operatorname{ext} X}$. This proves the theorem.

I would like to thank the referee for his very useful suggestions.

\section{REFERENCES}

1. H. Bauer and H. S. Bear, The part metric in convex sets, Pacific J. Math. 30 (1969), 15-33. MR 43 \#859.

2. R. D. Bourgin, Barycenters of measures on certain noncompact convex sets, Trans. Amer. Math. Soc. 154 (1971), 323-340. MR 42 \#6582.

3. G. Choquet, Lectures on analysis. Vols. I, II, III, Benjamin, New York, 1969. MR $40 \# 3252 ; \# 3253$; \#3254.

4. N. Dunford and J. T. Schwartz, Linear operators. I: General theory, Pure and Appl. Math., vol. 7, Interscience, New York, 1958. MR 22 \#8302.

5. J. L. Kelley, General topology, Van Nostrand, Princeton, N. J., 1955. MR 16, 1136.

6. S. S. Khurana, Measures and barycenters of measures on convex sets in locally convex spaces. I, II, J. Math. Anal. Appl. 27 (1969), 103-115; ibid. 28 (1969), 222-229. MR $39 \# 4631$; \#6042. 
7. S. S. Khurana, Characterization of extreme points, J. London Math. Soc. (2) 5 (1972), 102-104.

8. - Barycenters, extreme points, and strongly extreme points, Math. Ann. 198 (1972), 81-84.

9. J. D. Knowles, Measures on topological spaces, Proc. London Math. Soc. (3) 17 (1967), 139-156. MR 34 \#4441.

10. R. R. Phelps, Lectures on Choquet theorem, Van Nostrand, Princeton, N. J., 1966. MR 33 \#1690.

11. J. D. Pryce, On the representation and some separation properties of semiextremal subsets of convex sets, Quart. J. Math. Oxford Ser. (2) 20 (1969), 367-382. MR $40 \# 4719$.

12. M. S. Rieffel, Dentable subsets of Banach spaces with applications to a RadonNikodyn theorem, Functional Analysis Proc. Conf. (Univ. of California, Irvine), Thompson Book, Washington, D. C., 1967, pp. 71-77. MR 36 \#5668.

13. H. H. Schaeffer, Topological vector spaces, Macmillan, New York, 1966. MR 33 \#1689.

14. D. Sondermann, Masse auf lokalbeschränkten Räumen, Ann. Inst. Fourier (Grenoble) 19 (1970), fasc. 2, 33-113. MR 41 \#5587.

15. F. Topsфe, Measure and topology, Lecture Notes in Math., vol. 133, SpringerVerlag, New York, 1970.

16. V. S. Varadarajan, Measures on topological spaces, Mat. Sb. 55 (97) (1961), 35-100; English transl., Amer. Math. Soc. Transl. (2) 48 (1965), 161-220. MR 26 \#6342.

DEPARTMENT OF MATHEMATICS, UNIVERSITY OF IOWA, IOWA CITY, IOWA 52240 